University of Nebraska - Lincoln DigitalCommons@University of Nebraska - Lincoln

Civil Engineering Faculty Publications

Civil Engineering

2012

\title{
Optimal Advance Detector Location for Green Termination Systems on High-Speed Isolated Rural Intersections
}

Lili Du

Purdue University

Anuj Sharma

University of Nebraska - Lincoln, anujs@iastate.edu

Srinivas Peeta

Purdue University, peeta@purdue.edu

Follow this and additional works at: http://digitalcommons.unl.edu/civilengfacpub

Part of the Civil Engineering Commons, and the Other Civil and Environmental Engineering Commons

Du, Lili; Sharma, Anuj; and Peeta, Srinivas, "Optimal Advance Detector Location for Green Termination Systems on High-Speed Isolated Rural Intersections" (2012). Civil Engineering Faculty Publications. 52.

http://digitalcommons.unl.edu/civilengfacpub/52

This Article is brought to you for free and open access by the Civil Engineering at DigitalCommons@University of Nebraska - Lincoln. It has been accepted for inclusion in Civil Engineering Faculty Publications by an authorized administrator of DigitalCommons@University of Nebraska - Lincoln. 


\title{
Optimal Advance Detector Location for Green Termination Systems on High-speed Isolated Rural Intersections
}

\author{
Lili Du ${ }^{\mathrm{a}}$, Anuj Sharma ${ }^{\mathrm{b}}$, Srinivas Peeta ${ }^{\mathrm{c}^{*}}$ \\ ${ }^{a}$ NEXTRANS Center, Purdue University, West Lafayette, IN 47906 \\ ${ }^{b}$ Department of Civil Engineering, University of Nebraska-Lincoln, Lincoln, NE 68588 \\ ${ }^{c}$ School of Civil Engineering, Purdue University, West Lafayette, IN 47907-2051
}

\begin{abstract}
This study seeks to identify the optimal location of an advance point traffic detector (APTD) to support green termination algorithms for enhanced dilemma zone protection systems at high-speed isolated signalized rural intersections. This is done by developing a nonlinear optimization model with the objective to maximize the opportunities to predict empty dilemma zones during a look-ahead time period subject to the prediction accuracy which is manifested through prediction efficiency and safety constraints in the model. The distance of the APTD from the stop bar of the intersection represents the decision variable. The Golden-Section line search algorithm combined with numerical integration techniques is proposed to identify the feasible region and the optimal solution. The proposed methodology is analyzed using field data from a high-speed isolated intersection in Lincoln, Nebraska. The numerical experiments demonstrate that as the constraints associated with prediction efficiency and safety are relaxed, the feasible range to deploy the APTD increases. The optimal solution is influenced by the relationship between the prediction error and the location of the APTD, illustrating the need to robustly calibrate the function used to estimate the variance of the prediction error using field data. From a practice standpoint, the study confirms the potential concerns related to the performance efficiency of green termination systems using a point detector; typical field implementations locate the detector $750 \mathrm{ft}$ - $1000 \mathrm{ft}$ from the stop bar, which can potentially lead to significant levels of missed opportunities to terminate green safely. Overall, the proposed approach not only provides a systematic analytical methodology to determine the optimal location of the advance detector, but also to identify its feasible range based on user-specified thresholds related to efficiency and safety.
\end{abstract}

* Corresponding author. Tel: (765) 494-2209. E-mail address: peeta@ purdue.edu 


\title{
Optimal Advance Detector Location for Green Termination Systems on High-speed Isolated Rural Intersections
}

\author{
Lili Du, Anuj Sharma, Srinivas Peeta
}

\section{Background and motivation}

In contrast to urban intersections with balanced traffic for main and cross streets, rural intersections typically have significantly lower volume to saturation flow rate for the cross street but relatively congested traffic for the main street. Therefore, traffic signal control algorithms for high speed isolated rural intersections differ from that of a typical urban intersection control. Under the conditions of unbalanced traffic flow at rural intersections, it is advantageous to continue the green phase on the main street until some vehicles arrive on the cross street to justify the interruption of main street traffic (Newell, 1989). It is possible for the main street queue to clear before any vehicle demands the cross-street green. Under such situations, high speed traffic on the main street may face a yellow phase. At the onset of the yellow phase, a driver approaching the intersection on the main street has to decide whether to stop or go. A risky decision to go can possibly lead to a right angle crash, and a risky decision to stop can possibly lead to a severe rearend crash. The area in which the driver has a high risk of making a wrong decision is referred to as the decision dilemma zone (Olson and Rothery, 1962; May,1968; Parsonson, 1978). Bonneson et al. (1994) indicate that the start of the dilemma zone is 5-6 seconds upstream and the end is about 3 seconds upstream of the stop bar. The proposed study uses this commonly applied dilemma zone boundary in the temporal domain (5.5 seconds - 3 seconds).

Dilemma zone protection systems are deployed to operate rural isolated high speed signalized intersections to provide safe and efficient operations (Bonneson et al., 2002; Sharma et al., 2011b). There are two basic algorithms used by dilemma zone protection systems: the green extension algorithm and the green termination algorithm. In the green extension algorithm (Zegeer, 1977; Sharma et al., 2007), the green phase of the high-speed approach is extended until there is no vehicle in the dilemma zone. However, an upper threshold, a maximum green time, is provided for this operation to avoid excessive delays to the cross street traffic. The termination of green on reaching this upper threshold is defined as max-out. As the number of lanes increases, the probability of finding an empty dilemma zone within the maximum green time decreases. This problem becomes worse when the high speed approach carries medium-to-heavy traffic volumes. Also, the safety benefits are negated when the high speed through phase is arbitrarily terminated on reaching the maximum green time. Sharma et al. (2006) provide a detailed analysis of this problem. For the intersection analyzed in their study, the implementation of the green extension logic led 
to a max-out in 3.5 to 40 percent of the cycles per hour during the peak traffic flow periods, and around 200 dilemma zone incursions per day.

Green termination algorithms (Kronborg et al., 1997; Bonneson et al., 2002; Zimmerman et al., 2003; Zimmerman, 2007) have been developed in recent years to address the limitations of green extension algorithms. They search for an optimal time to terminate green beyond the minimum green time. A combination of cross-street delays and number of vehicles in the dilemma zone is used to determine the optimal green termination time. There is a high cost of safety associated with the presence of vehicles in their dilemma zone at the end of green, as they have higher risk of crash. Hence, one of the objectives of the green termination logic is to minimize this cost by identifying green termination points with empty dilemma zones. Thus, it is critical to accurately predict the opportunities for having empty dilemma zones. An advance detector upstream of the stop bar is placed on the high-speed through approach for predicting the number of vehicles in the dilemma zone in the near-term future (which is labeled the "look-ahead time period" in Fig. 1). The performance of the signal is evaluated assuming that green is to be terminated at any point in the current look-ahead time period. Based on the evaluation of intersection performance in this look-ahead time period, if the current time is the best time to end the green, the green phase is terminated; otherwise the green phase is continued. If the green is not terminated at the current instance, the search process for the optimal green termination time is repeated after a small wait time (usually 0.5 seconds).

As illustrated in Fig. 1, the advance detector in green termination systems is located at some distance in advance of the dilemma zone boundary (DLZ Start) of the fastest vehicle that is to be protected. The length of the look-ahead time period is the time it takes the fastest vehicle being protected to traverse the distance from the location of the advance detector to the start of its dilemma zone boundary (Bonneson, 2002; Zimmerman et al., 2003; Zimmerman, 2007). On the left side of Fig. 1, a high-speed approach is shown with an advance detector located at 800 feet from the stop bar. The dilemma zone boundaries extend from $444 \mathrm{ft}$ to $242 \mathrm{ft}$ ( 5.5 seconds - 3 seconds in time domain) for a vehicle traveling at $55 \mathrm{mph}$. Time 0 represents the current time. Any event registered before the current time is the history and any event that will occur after time 0 is the future. On the right side of Fig. 1, the trajectories of three vehicles traveling at $55 \mathrm{mph}$ are illustrated. The events representing the three vehicles crossing the advance detector occur at times $t_{1}, t_{2}$, and $t_{3}$. These events are the history and have been recorded prior to the current time $(t=0)$.

The estimated arrival times of the three vehicles to their dilemma zones, denoted by $\widehat{t_{1}^{\widehat{ }}}, \widehat{t_{2}^{\natural n}}$, and $\widehat{t_{3}^{n n}}$, can be predicted by assuming that these vehicles will maintain their speeds measured at the advance detector. Any vehicle arriving after the current time and traveling at a speed lower than $55 \mathrm{mph}$ will not affect the number of vehicles estimated in the look-ahead time period. The duration of the look-ahead time period can be increased by shifting the advance detector further upstream. 
There are two countervailing aspects that need to be considered in determining the advance detector location. First, the length of the look-ahead time period increases in proportion to the distance of the advance detector from the stop bar, thus increasing the probability of finding a time point at which the dilemma zone will be empty. Second, the accuracy of the prediction of an empty dilemma zone decreases in proportion to the distance of the advance detector from the stop bar, as the vehicles have more temporal opportunities to change their speeds after they pass the advance detector. The need to trade-off these two aspects represents the conceptual platform in this study to formulate a systematic mathematical model to identify the optimal location of the advance detector.

Prior studies (Bonneson et al., 2002; Sharma et al., 2011b) have highlighted the problem of speedchange behavior affecting the prediction of empty dilemma zones. Bonneson et al. (2002) reported that of 104 observations, $82 \%$ of the vehicles changed speed while traveling from the upstream location to the stop bar. Nearly $50 \%$ of the vehicles had over $2 \mathrm{mph}$ change in speeds. For Intelligent Detection-Control Systems, they proposed to use conservative dilemma zone boundaries of 1.5 to 6.5 seconds (instead of 2.55.5 seconds) in the field to minimize the impacts of speed changes. The adoption of conservative dilemma zone boundaries can significantly reduce the number of opportunities to find empty dilemma zones, thereby reducing both safety and efficiency of operations at the intersection (Sharma et al., 2006; Zimmerman, 2007). While speed prediction accuracies have been identified as a problem for green termination systems, to the best of our knowledge there exists no analytical formulation to provide bounds on prediction accuracy with the change in the location of the advance detector. This paper proposes techniques to identify these bounds and find the optimal location to place the advance detector such that the prediction error is within a limit that can be specified by the traffic engineer.

The proposed approach to determine the optimal advance detector location and analyze its effectiveness is briefly summarized hereafter. First, a mathematical formulation is provided to predict the number of empty dilemma zones in a look-ahead time period. In conjunction with this formulation, a nonlinear optimization model is proposed to find the optimal location to deploy the advance detector in dilemma zone protection systems. The solution method is then described. Numerical experiments are conducted in which the field traffic data are used to calibrate the probability density functions of the random variables and the related parameters for the nonlinear optimization model. The experiment results indicate that the methodology identifies the minimum and maximum distances as well as the optimal location to deploy the ATPD with the desired prediction accuracy requirements. Hence, the proposed methodology provides a systematic analytical approach to implement the associated dilemma zone protection systems in practice.

The remainder of the paper is organized as follows. Section 2 formulates the problem and develops the solution methodology. Section 3 conducts numerical experiments to implement the proposed 3 
methodology based on the field data from Lincoln, Nebraska. It discusses the associated results and related insights. Section 4 provides some concluding comments.

\section{Problem formulation and solution methodology}

This section first describes the problem and its characteristics. Then, it introduces a mathematical formulation to predict an empty dilemma zone based on the traffic data at the APTD. It then develops the formulations to determine the probability: (i) that the dilemma zone is empty in the field, (ii) that the dilemma zone is predicted empty, and (iii) of the intersection of (i) and (ii). Next, a nonlinear optimization model is proposed for the optimal location of the APTD. This is followed by a line search algorithm to solve the model.

\subsection{Problem description and characteristics}

This study seeks to determine the optimal location of the advance detector so as to maximize the number of predicted empty dilemma zones over a look-ahead time period subject to constraints on the prediction accuracy. The underlying objective is that the larger the number of empty dilemma zones, the higher the number of potential opportunities to efficiently terminate the green time while ensuring safety and mobility needs at the intersection. These needs are manifested through the prediction accuracy associated with the empty dilemma zones. The prediction accuracy is represented through the prediction efficiency and the prediction safety. That is, the higher these values, the higher the prediction accuracy. To define the prediction efficiency and the prediction safety, we first illustrate the linkage between the empty dilemma zone prediction and the operational performance of the dilemma zone protection system at an intersection in Table 1. As defined in the table, the prediction efficiency is reflected through the need to reduce the error event $e^{e}$ that the dilemma zone is predicted occupied while empty in the field. That is, the larger the number of events $e^{e}$, the greater the number of potential missed opportunities to terminate the green time. The prediction safety is viewed through the need to reduce the error event $e^{s}$ that the dilemma zone is predicted empty though it is occupied in the field. The error event $e^{s}$ has safety implications for the intersection as it may cause the green light termination while vehicles are in their dilemma zone. Hence, to ensure acceptable levels of operational efficiency and safety at the intersection, there is a need to constrain the occurrences of the events $e^{e}$ and $e^{s}$.

The scope of the problem in developing the mathematical model is characterized as follows. The spatial area of interest in terms of the traffic events lies between the APTD location and the stop bar at the intersection. The advance detector provides data on the time stamp of the vehicle detection and its speed. The empty dilemma zone prediction is performed after each time a vehicle passes through the advance detector. While the study does not constrain that two consecutive vehicles must be on the same lane of an 
approach, it does not cover the situation that two vehicles in adjacent lanes arrive at the detector together with the same speed; that is, the model determines a single dilemma zone. Further, the model does not consider situations involving overtaking between vehicles downstream of the APTD location.

\subsection{Prediction of empty dilemma zone and related events}

We first observe the movements of two vehicles which consecutively pass through the study area from the APTD to the stop bar in the field. Let vehicle $c_{2}$ pass through the advance detector after vehicle $c_{1}$; we label vehicle $c_{1}$ as the "head" vehicle and vehicle $c_{2}$ as the "following" vehicle. Then, an empty dilemma zone occurs between these two consecutive vehicles if the vehicle $c_{2}$ arrives at its dilemma zone later than its head vehicle $c_{1}$ leaves its dilemma zone. Hence, to predict an empty dilemma zone, we need to first determine the time stamp that vehicle $c_{1}$ leaves its dilemma zone, $t_{1}^{\text {out }}$, and the time stamp that the vehicle $c_{2}$ enters its dilemma zone, $t_{2}^{\text {in }}$. Ideally, $t_{1}^{\text {out }}$ and $t_{2}^{\text {in }}$ can be determined precisely if the variation in vehicle speeds after they pass through the APTD can be accurately measured. However, this is not realistic in the field due to the dynamic nature of traffic and the absence of sensors to do so. Consequently, as illustrated in Fig. 2, this study approximates $t_{1}^{\text {out }}$ and $t_{2}^{\text {in }}$ using Equations (1) and (2), respectively, by assuming that the individual vehicles maintain their speeds after they pass through the APTD, and that a dilemma zone starts at 5.5 seconds and ends at 3.0 seconds upstream of the stop bar.

$$
\begin{aligned}
& \widehat{t_{1}^{\widehat{o u}} t}=t_{1}+\frac{\left(y-3 v_{1}\right)}{v_{1}} \\
& \widehat{t_{2}^{\text {m }}}=t_{2}+\frac{\left(y-5.5 v_{2}\right)}{v_{2}}
\end{aligned}
$$

Hence, $t_{1}^{\widehat{o u} t}$ represents the prediction of $t_{1}^{\text {out }}$ and $\widehat{t_{2}^{\mathrm{mn}}}$ represents the prediction of $t_{2}^{i n} \cdot t_{1}$ and $t_{2}$ represent the observed time stamps that the vehicles $c_{1}$ and $c_{2}$ pass through the APTD, respectively, and $v_{1}$ and $v_{2}$ represent their measured speeds at the ATPD, respectively. $y$ is the distance of the ATPD from the stop bar. Accordingly, the event $\hat{E}$ that the dilemma zone is predicted to be empty can be mathematically described by Equation (3):

$$
\widehat{E}: \widehat{t_{2}^{\mathrm{nn}}}-\widehat{t_{1}^{\widehat{o u}} t}=t_{2}-t_{1}+\frac{\left(y-5.5 v_{2}\right)}{v_{2}}-\frac{\left(y-3 v_{1}\right)}{v_{1}}=\mathrm{H}-2.5+\frac{y}{v_{2}}-\frac{y}{v_{1}} \geq 0
$$

where $H=t_{2}-t_{1}$ is the observed time headway between the two consecutive vehicles at the location of the APTD.

Based on the field data, the speeds of the individual vehicles and the headways between vehicles at the APTD vary according to certain probability distributions. Hence, $H, v_{1}$ and $v_{2}$ at the APTD are treated as random variables. Then, the event $\hat{E}$ occurs with the probability represented by Equation (4): 


$$
P(\widehat{E})=P\left(H-2.5+\frac{y}{v_{2}}-\frac{y}{v_{1}} \geq 0\right)=\iiint_{H-2.5+\frac{y}{v_{2}}-\frac{y}{v_{1}} \geq 0} f\left(H, v_{1}, v_{2}\right) d v_{1} d v_{2} d H
$$

where $f\left(H, v_{1}, v_{2}\right)$ represents the joint probability density of $H, v_{2}$, and $v_{1}$. The expression for $f\left(H, v_{1}, v_{2}\right)$ can be obtained from the density functions for headway and speed in the literature (Zhang et al., 2007; Gerlough and Huber, 1975). Accordingly, $P(\widehat{E})$ is a triple integral of $f\left(H, v_{1}, v_{2}\right)$ over the half space defined by $\left(H-2.5+\frac{y}{v_{2}}-\frac{y}{v_{1}}\right) \geq 0$. We provide a more detailed discussion on $f\left(H, v_{1}, v_{2}\right)$ in Section 2.4, and demonstrate the process to specify $f\left(H, v_{1}, v_{2}\right)$ in the experiments in Section 3.

Next, extending the conceptual representation of the event $\hat{E}$, the event $E$ that the dilemma zone is empty in the field and its probability $P(E)$ are analytically derived hereafter. The predicted values of $t_{1}^{\text {out }}$ and $\widehat{t_{2}^{\natural n}}$ in Equations (1) and (2) are influenced by two potential sources of random error, the speed variation of vehicles downstream of the APTD and the possible measurement error of the vehicle arrival time at the APTD. These errors are denoted by the random variables $\varepsilon_{1}^{\text {out }}$ and $\varepsilon_{2}^{\text {in }}$. Based on these error terms, the linear functions in Equations (5) and (6) are used to represent the observed time stamps of $t_{1}^{\text {out }}$ and $t_{2}^{\text {in }}$, respectively.

$$
\begin{aligned}
& t_{1}^{\text {out }}=t_{1}^{\widehat{\text { ou }} t}+\varepsilon_{1}^{\text {out }}=t_{1}+\frac{\left(y-3 v_{1}\right)}{v_{1}}+\varepsilon_{1}^{\text {out }} \\
& t_{2}^{i n}=t_{2}^{\widehat{t n}}+\varepsilon_{2}^{i n}=t_{2}+\frac{\left(y-5.5 v_{2}\right)}{v_{2}}+\varepsilon_{2}^{i n}
\end{aligned}
$$

Following Equation (3), Equation (7) mathematically describes the event $E$ that the dilemma zone is empty in the field:

$$
E: t_{2}^{\text {in }}-t_{1}^{\text {out }} \geq 0 \Leftrightarrow \varepsilon_{2}^{\text {in }}-\varepsilon_{1}^{\text {out }}+\left(H-2.5+\frac{y}{v_{2}}-\frac{y}{v_{1}}\right) \geq 0
$$

Accordingly, Equation (8) represents the probability that event $E$ occurs in a look-ahead time period:

$$
P(E)=P\left(\left[\varepsilon+\left(H-2.5+\frac{y}{v_{2}}-\frac{y}{v_{1}}\right) \geq 0\right]\right)
$$

where $\varepsilon=\varepsilon_{2}^{\text {in }}-\varepsilon_{1}^{\text {out }}$ represents the empty dilemma zone prediction error; also referred to as "prediction error" in the remainder of this paper. As $H, v_{2}, v_{1}$, and $\varepsilon$ are continuous random variables, $P(E)$ can be computed using the integral in Equation (9), where $\gamma\left(H, v_{1}, v_{2}, \varepsilon\right)$ represents the joint probability density function of , $v_{2}, v_{1}$, and $\varepsilon$.

$$
P(E)=\iiint_{\varepsilon+\left(H-2.5+\frac{y}{v_{2}}-\frac{y}{v_{1}}\right) \geq 0} \gamma\left(H, v_{1}, v_{2}, \varepsilon\right) d \varepsilon d v_{1} d v_{2} d H
$$

As $\varepsilon$ is a function of $\varepsilon_{1}^{\text {out }}$ and $\varepsilon_{2}^{\text {in }}$, it is also characterized by different sources of random error. Hence, it is considered to be independent of $v_{1}, v_{2}$, and $H$. Then, we have $\gamma\left(H, v_{2}, v_{1}, \varepsilon\right)=\rho(\varepsilon) f\left(H, v_{2}, v_{1}\right)$, where 
$\rho(\varepsilon)$ represents the probability density function of $\varepsilon$. Accordingly, Equation (9) is equivalent to Equation (10), where $s\left(v_{1}, v_{2}, H\right)$ represents the region defined by the feasible ranges of $v_{1}, v_{2}$, and $H$; that is, $v_{1} \in$ $(0,+\infty), v_{2} \in(0,+\infty)$, and $H \in(0,+\infty)$.

$$
P(E)=\iiint_{s\left(v_{1}, v_{2}, H\right)}\left(\int_{\varepsilon \geq-\left(H-2.5+\frac{y}{v_{2}}-\frac{y}{v_{1}}\right)}^{+\infty} \rho(\varepsilon) d \varepsilon\right) f\left(H, v_{1}, v_{2}\right) d v_{1} d v_{2} d H
$$

Substituting $\left(\int_{\varepsilon \geq-\left(H-2.5+\frac{y}{v_{2}}-\frac{y}{v_{1}}\right)}^{+\infty} \rho(\varepsilon) d \varepsilon\right)$ in Equation (10) by $P\left(\varepsilon \geq-\left(H-2.5+\frac{y}{v_{2}}-\frac{y}{v_{1}}\right)\right)$, we obtain Equation (11) to represent $P(E)$, which is equal to the triple integral of $P\left(\varepsilon \geq-\left(H-2.5+\frac{y}{v_{2}}-\right.\right.$ $\left.\left.\frac{y}{v_{1}}\right)\right) f\left(v_{1}, v_{2}, H\right)$ over the feasible region defined by $s\left(v_{1}, v_{2}, H\right)$.

$$
P(E)=\iiint_{s\left(v_{1}, v_{2}, H\right)} P\left(\varepsilon \geq-\left(H-2.5+\frac{y}{v_{2}}-\frac{y}{v_{1}}\right)\right) f\left(v_{1}, v_{2}, H\right) d v_{1} d v_{2} d H
$$

Further discussion on the distribution of $\varepsilon$ is provided in Section 2.4, and the computation of $P\left(\varepsilon \geq-\left(H-2.5+\frac{y}{v_{2}}-\frac{y}{v_{1}}\right)\right)$ is illustrated in Section 3 using the collected field data in the study experiments.

Equation (11) seems to indicate that $P(E)$ is a function of the location of the APTD, $y$. However, in reality, the unfolding traffic flow determines whether a dilemma zone is empty or not at a given time, independent of the location of the advance detector. That is, an empty dilemma zone exists regardless of the location of the detector. However, the location of the APTD influences the observed traffic data, the prediction error, and the associated distributions. Therefore, for the traffic flow that has unfolded at an intersection, the value of $P(E)$ is fixed and independent of $y$. This aspect is validated in the experiments in Section 3.3.2. Here, we provide a brief conceptual linkage to the experimental validation by first noting that the location of the APTD influences the observed values of $v_{1}, v_{2}$, and $H$ and the unobserved value of $\varepsilon$. Hence, the value of $y$ affects the distributions of $H, v_{1}, v_{2}$, and $\varepsilon$. Therefore, the value of $y$ influences the distribution of $\varepsilon$ in Equation (11). In the experiments, the collected field data are used to determine the distributions of $\varepsilon$ for different values of $y$, and the $\varepsilon$ distribution corresponding to each $y$ value is used to compute $P(E)$ using Equation (11). The computed values of $P(E)$ are practically identical, thereby validating the relationship between $y$ and the estimation of $P(E)$ using Equation (11).

Using conceptual and mathematical processes similar to those for deriving $P(\hat{E})$ and $P(E)$, Equation (12) derives the joint probability that the dilemma zone is empty in the field and is also predicted empty. 


$$
\begin{aligned}
& P(E \hat{E})=P\left(\left[\varepsilon \geq-\left(H-2.5+\frac{y}{v_{2}}-\frac{y}{v_{1}}\right)\right],\left[H-2.5+\frac{y}{v_{2}}-\frac{y}{v_{1}} \geq 0\right]\right) \\
& =\iiint \int_{\varepsilon \geq-\left(H-2.5+\frac{y}{v_{2}}-\frac{y}{v_{1}}\right), H-2.5+\frac{y}{v_{2}}-\frac{y}{v_{1}} \geq 0} \gamma\left(H, v_{1}, v_{2}, \varepsilon\right) d \varepsilon d v_{1} d v_{2} d H \\
& =\iiint_{H-2.5+\frac{y}{v_{2}}-\frac{y}{v_{1}} \geq 0} \int_{\varepsilon \geq-\left(H-2.5+\frac{y}{v_{2}}-\frac{y}{v_{1}}\right)} \rho(\varepsilon) d \varepsilon f\left(H, v_{1}, v_{2}\right) d v_{1} d v_{2} d H \\
& =\iiint_{H-2.5+\frac{y}{v_{2}}-\frac{y}{v_{1}} \geq 0} P\left(\varepsilon \geq-\left(H-2.5+\frac{y}{v_{2}}-\frac{y}{v_{1}}\right)\right) f\left(v_{1}, v_{2}, H\right) d v_{1} d v_{2} d H
\end{aligned}
$$

Mathematically, it is equal to the triple integral of $P\left(\varepsilon \geq-\left(H-2.5+\frac{y}{v_{2}}-\frac{y}{v_{1}}\right)\right) f\left(v_{1}, v_{2}, H\right)$ over the half space defined by $H-2.5+\frac{y}{v_{2}}-\frac{y}{v_{1}} \geq 0$. The expressions for $P(E), P(\widehat{E})$ and $P(E \hat{E})$ are used in the next section to develop the nonlinear optimization model to identify the optimal APTD location in dilemma zone protection systems.

\subsection{Optimization model}

The optimization model to determine the optimal location to deploy the APTD seeks to maximize the expected number of times that a dilemma zone is predicted empty in a look-ahead time period while satisfying the prediction efficiency and safety requirements. This objective function is established as follows. Using $v_{85}$, the $85^{\text {th }}$ percentile speed based on the field data, as the highest speed of individual vehicles to be protected using dilemma zone protection systems, the length of a look-ahead time period is $\left(\frac{y-5.5 v_{85}}{v_{85}}\right)$. Note that without loss of generality, a different value can be used for the highest speed if desired by the traffic engineer. Let $\bar{H}$ denote the average headway in the look-ahead time period at the APTD. Then, the system has an average number of $\left(\frac{y-5.5 v_{85}}{v_{85} \bar{H}}\right)$ opportunities to predict if the dilemma zone is empty or not as individual vehicles pass through the APTD. Also, Equation (4) provides the predicted probability of detecting an empty $(P(\widehat{E}))$ or non-empty $(1-P(\widehat{E}))$ dilemma zone as each vehicle passes through the APTD in a look-ahead time period. Viewing each prediction opportunity in a look-ahead time period as an independent instance with two possible outcomes that have constant probabilities, the resulting Bernoulli process to capture the probability of the number of empty dilemma zones in that look-ahead time period follows a Binomial distribution. Accordingly, the mean of this Binomial distribution represents the expected number of times that the dilemma zone is predicted empty during a look-ahead time period. This is illustrated in Equation (13), which represents the objective function of the proposed nonlinear optimization model. 


$$
\frac{y-5.5 v_{85}}{v_{85} \bar{H}} P(\hat{E})
$$

As discussed in Section 2.1, however, this objective is constrained by the need to ensure acceptable levels of prediction efficiency and prediction safety. In this study, the prediction efficiency $p_{e}$ is defined as the probability of the event that the dilemma zone is predicted empty given that it is empty in the field. To sustain an acceptable value of $p_{e}$ for dilemma zone protection systems, we require its complement, the probability of the error event $e^{e}=(\bar{E} \mid E)$, to be less than a user-defined efficiency-related threshold parameter $\beta$. This leads to Equation (14), which is used as the prediction efficiency constraint in the optimization model.

$$
p_{e}=P(\hat{E} \mid E) \geq 1-\beta \Leftrightarrow \frac{P(\hat{E} E)}{P(E)} \geq 1-\beta
$$

Similarly, the prediction safety $p_{s}$ is defined as the probability of the event that the dilemma zone is predicted occupied given that it is occupied in the field. To ensure an acceptable level for $p_{s}$, we require that its complement, the probability of the error event $e^{s}=(\hat{E} \mid \bar{E})$, to be less than a user-defined safetyrelated threshold parameter $\alpha$. Equation (15) represents this prediction safety constraint in the proposed optimization model.

$$
p_{s}=P(\overline{\hat{E}} \mid \bar{E})=1-P(\hat{E} \mid \bar{E})=1-\frac{P(\widehat{E} \bar{E})}{P(\bar{E})}=1-\frac{P(\widehat{E})-P(\widehat{E} E)}{P(\bar{E})} \geq 1-\alpha
$$

As can be noted, the LHS of the final forms of Equations (14) and (15) can be computed using known probability results illustrated in Section 2.2.

Finally, the non-negativity of $y$ is represented by constraint (16).

$$
y \geq 0
$$

Using the objective function (13) along with constraints (14), (15) and (16), and treating the location of the APTD $y$ as the decision variable, we obtain the nonlinear optimization model labeled $M 1$.

Model $M 1$

$$
\begin{array}{ll}
\text { Max } & \frac{y-5.5 v_{85}}{v_{85} \bar{H}} P(\hat{E}) \\
\text { s.t. } & p_{e}=P(\hat{E} \mid E)=\frac{P(\widehat{E} E)}{P(E)} \geq 1-\beta \\
& p_{s}=1-P(\hat{E} \mid \bar{E})=1-\frac{P(\hat{E} \bar{E})}{P(\bar{E})}=\frac{P(\hat{E})-P(\hat{E} E)}{P(\bar{E})} \geq 1-\alpha \\
& y \geq 0
\end{array}
$$


The user-defined efficiency- and safety-related thresholds $\beta$ and $\alpha$, respectively, can be treated as input parameters in $M 1$. The traffic engineer (or system operator) may provide these threshold values based on the requirements of efficiency and safety for the dilemma zone protection systems at the relevant intersection. Another option is to explore different combinations of $\beta$ and $\alpha$ in $M 1$. The model determines the corresponding optimal locations to deploy the ATPD. Then, the system operator can choose the desired combination satisfying their requirements related to efficiency and safety.

Substituting the expressions for $P(\hat{E}), P(E)$, and $P(\widehat{E} E)$ from Equations (4), (11), and (12), respectively, into $M 1$, we obtain model $M 2$.

Model M2

$\mathrm{M}$

$$
\begin{gathered}
\operatorname{Max} \frac{y-5.5 v_{85}}{v_{85} \bar{H}} \iiint_{H-2.5+\frac{y}{v_{2}}-\frac{y}{v_{1}} \geq 0} f\left(H, v_{1}, v_{2}\right) d v_{1} d v_{2} d H \\
\iiint_{H-2.5+\frac{y}{v_{2}}-\frac{y}{v_{1}} \geq 0} P\left(\varepsilon \geq-\left(H-2.5+\frac{y}{v_{2}}-\frac{y}{v_{1}}\right)\right) f\left(H, v_{1}, v_{2}\right) d v_{1} d v_{2} d H \\
\hline
\end{gathered}
$$

s.t.

$$
\begin{aligned}
& \iiint_{S\left(v_{1}, v_{2}, H\right)} P\left(\varepsilon \geq-\left(H-2.5+\frac{y}{v_{2}}-\frac{y}{v_{1}}\right)\right) f\left(H, v_{1}, v_{2}\right) d v_{1} d v_{2} d H \\
& \geq(1-\beta) \\
& \frac{\iiint_{H-2.5+\frac{y}{v_{2}}-\frac{y}{v_{1}} \geq 0} P\left(\varepsilon \leq-\left(H-2.5+\frac{y}{v_{2}}-\frac{y}{v_{1}}\right)\right) f\left(H, v_{1}, v_{2}\right) d v_{1} d v_{2} d H}{1-\iiint_{S\left(v_{1}, v_{2}, H\right)} P\left(\varepsilon \geq-\left(H-2.5+\frac{y}{v_{2}}-\frac{y}{v_{1}}\right)\right) f\left(H, v_{1}, v_{2}\right) d v_{1} d v_{2} d H} \leq \alpha \\
& y \geq 0 \\
& v_{1}, v_{2} \text {, and } H \text { are random variables; } s\left(v_{1}, v_{2}, H\right) \text { is the feasible range of } v_{1}, v_{2}, H \text {. }
\end{aligned}
$$

\subsection{Solution method}

Model M2 is a nonlinear program with a single variable. As the objective function and the constraints are intricate expressions, a customized line search algorithm combined with numerical integral techniques is used to derive the local optimal solution. The solution method includes the following four main steps.

First, the distributions of the random variables $H, v_{1}$ and $v_{2}$ and the correlations among them are identified to obtain the joint density function $f\left(H, v_{1}, v_{2}\right)$ in model $M 2$. We use the findings of past studies for the distributions; $H$ is usually considered to follow a log-normal distribution (Zhang et al., 2007), and speeds $v_{1}$ and $v_{2}$ are usually treated as normal distributions (Gerlough and Huber, 1975). In a deployment context, the parameters of these distributions can be calibrated using field data. By contrast, the correlations between $H, v_{1}$ and $v_{2}$ at the APTD have not been adequately explored in the literature. In this study, we 
postulate that these correlations are, at best, weak based on the characteristics of high-speed isolated intersections in relation to the problem objective of capturing empty dilemma zones for green termination, as explained hereafter. The exploration of opportunities for empty dilemma zones in rural high-speed isolated intersections for green termination is not typically based on the traffic conditions immediately after the initiation of green on the main approach when potentially queued vehicles are being discharged. Rather, the period of interest is when vehicles approach the intersection more infrequently as the green phase continues. That is, the average headway between vehicles is sufficiently larger than the typical headways when car-following type behavior is strong. Under these conditions, the speeds of the two consecutive vehicles have little, if any, correlation between them arising from driver psychology, and consequently, with their headway as well. The potential dependency between speeds is further weakened by the fact that the measurements of the speeds $v_{1}$ and $v_{2}$ occur at a single spatial point, the APTD location, but at a time latency equal to the headway $H$. Hence, as the headway increases, the dependency between speeds measured at different time stamps also reduces. In summary, we assume that $H, v_{1}$ and $v_{2}$ are weakly correlated and approximate $f\left(H, v_{1}, v_{2}\right)$ by the product of their individual distributions. This assumption is validated in Section 3 for the field data used in the study experiments. However, if the field data at an intersection show that $H, v_{1}$ and $v_{2}$ are strongly dependent/correlated, their joint probability density function $f\left(H, v_{1}, v_{2}\right)$ can be approximated using Copula techniques (Kumar, 2010).

Second, the closed-form expression for $P\left(\varepsilon \geq-\left(H-2.5+\frac{y}{v_{2}}-\frac{y}{v_{1}}\right)\right)$, denoted hereafter by $\psi$ for expository convenience, is determined. The determination of the expression for $\psi$ requires the distribution of $\varepsilon$. As $\varepsilon=\varepsilon_{2}^{\text {in }}-\varepsilon_{1}^{\text {out }}$, its distribution can be obtained based on the distributions of $\varepsilon_{2}^{\text {in }}$ and $\varepsilon_{1}^{\text {out }}$. To do so, we note that the process to develop Equations (5) and (6) in Section 2.2 indicates that $\varepsilon_{2}^{\text {in }}$ and $\varepsilon_{1}^{\text {out }}$ may be regarded as independent variables since the underlying prediction processes for $t_{1}^{\text {out }}$ and $\widehat{t_{2}^{m n}}$ are based on different vehicles at different times. Further, in the absence of systematic errors, there is no expectation of a bias (or skew) in either a positive or a negative direction for $\varepsilon_{1}^{\text {out }}$ and $\varepsilon_{2}^{\text {in }}$. Hence, without loss of generality, it is reasonable to assume that $\varepsilon_{1}^{\text {out }}$ and $\varepsilon_{2}^{\text {in }}$ follow identical normal distributions (that is, $\left.\varepsilon_{2}^{\text {in }} \sim N\left(\mu, \sigma^{2}\right), \varepsilon_{1}^{\text {out }} \sim N\left(\mu, \sigma^{2}\right)\right)$. Correspondingly, $\varepsilon=\varepsilon_{2}^{\text {in }}-\varepsilon_{1}^{\text {out }}$ obeys the normal distribution with the mean equal to zero and the variance equal to $2 \sigma^{2} ; \varepsilon \sim N\left(0,2 \sigma^{2}\right)$. Hence, the determination of the distribution of $\varepsilon$ requires the calibration of $\sigma^{2}$, the variance of $\varepsilon_{2}^{\text {in }}$ or $\varepsilon_{1}^{\text {out }}$. Bonneson et al. (2002) empirically illustrate that $\sigma^{2}$ is closely related to the advance detector location. Thereby, $\sigma^{2}$ can be calibrated using statistical methods based on field data. We use a linear regression function to estimate $\sigma^{2}$ in the experiments in Section 3. Further, as a closed-form expression does not exist for the cumulative 
probability of a normal distribution, a logistic approximation to the cumulative normal distribution (Lin, 1989 ) is used to represent $\psi$ in this study.

Third, the feasible region of $M 2$ is determined as follows. Using the closed-form expressions for $f\left(H, v_{1}, v_{2}\right)$ and $\psi$ from the previous two steps, the left hand sides of the prediction efficiency and safety constraints in Equations (18) and (19) are numerically calculated at each $y$. The feasible region of each constraint is identified by performing these calculations over the entire range of $y$ in small increments. The intersection of these feasible regions over the constraints represents the feasible region for model $M 2$. The feasible region is a line segment as $M 2$ has a single decision variable $y$. A key aspect in this context is that the left hand sides of the efficiency and safety constraints in Equations (18) and (19) include triple integrals. As their integrands are intricate, it is difficult to obtain a closed-form integral solution and further calculate the efficiency and safety constraints at each $y$. This can be addressed by using numerical integration techniques such as Monte Carlo algorithms (Press and Farrar, 1990) and Sparse Grids algorithms (Gerstner and Griebel, 1998) to compute the triple integral.

Finally, the local optimal solution of $M 2$ is determined. After the feasible region is determined in the previous step, solving the model $M 2$ implies finding the optimal solution for an unconstrained nonlinear programming problem on a one-dimensional line segment. Due to the intricate nature of the objective function in Equation (17), line search algorithms without derivatives, such as uniform search, dichotomous search, and the Golden-Section algorithm (Bazaraa, et al., 1993) can be used to find the local optimal solution. We use the Golden-Section algorithm in this study.

In summary, the solution method for $M 2$ consists of the following steps:

Step 1: Determine the joint density function $f\left(H, v_{1}, v_{2}\right)$.

Step 2: Determine the closed-form expression for $\psi$.

Step 3: Identify the feasible region of $M 2$.

Step 4: Obtain the local optimal solution for M2 using the Golden-Section algorithm.

Next, we demonstrate the implementation of the proposed solution method in practice.

\section{Numerical experiments}

This section implements the solution method for the optimization model $M 2$ and analyzes its performance using field data collected at an isolated high speed intersection in Lincoln, Nebraska. It first describes the field data collection setup. Then, it illustrates the steps of the solution method for the field data, including the calibration of $f\left(H, v_{1}, v_{2}\right)$, the computation of $\psi$, and the solution search process. Finally, the optimal solutions, sensitivity analyses, and associated insights are discussed. 


\subsection{Field data collection setup}

As shown in Fig. 3, the high-speed signalized intersection of Highway 2 and $84^{\text {th }}$ Street in Lincoln, Nebraska is used as the data collection site for the study experiments. Highway 2 is a major thoroughfare in Lincoln, particularly for heavy vehicles. The intersection approach of Highway 2 analyzed in this study has two through lanes, two left-turn lanes, and a right-turn lane. The site has a fusion of three radar-based sensors for monitoring all vehicles within $1,100 \mathrm{ft}$. of the intersection. Two video cameras monitor all vehicles in the vicinity of the intersection. The feed from the video is used for manual verification of data obtained from the radar sensors. The data from the radar sensor provide information on the date, time, identification number, range, and speed for each vehicle approaching the intersection. The data from the video is used to identify the vehicle type and lane information. Also, the signal phase indications are communicated from the signal cabinet " $C$ " to the pole-cabinet "A3" in Fig. 3, and logged in the radar sensor data file. This detection monitoring system is used to collect the trajectory data of 100 pairs of consecutive vehicles from 8:00am to12:00pm on a day, which represent the field data for the study experiments.

\subsection{Solution method implementation}

This section describes the implementation of the solution method for the collected field data.

\subsubsection{Calibration of $f\left(H, v_{1}, v_{2}\right)$}

As discussed in Section 2.4, the headway at the advance detector usually follows the log-normal distribution; let $H \sim \log ^{-} N\left(\omega, \eta^{2}\right)$. Similarly, the vehicle speeds $v_{1}$ and $v_{2}$ follow the normal distribution; $v_{1} \sim N\left(\tau_{1}, \theta_{1}{ }^{2}\right)$ and $v_{2} \sim N\left(\tau_{2}, \theta_{2}{ }^{2}\right)$. We calibrate the parameters $\tau_{1}, \tau_{2}, \theta_{1}, \theta_{2}, \omega$, and $\eta$ of the distributions of $H, v_{1}$ and $v_{2}$ using the field data. Section 2.2 indicates that the distributions of $H, v_{1}$ and $v_{2}$ depend on $y$. However, the collected field data suggests that these parameters are relatively stable with $y$ in the collected data range $460 \mathrm{ft}-1100 \mathrm{ft}$. Hence, we use constant distributions for $H, v_{1}$ and $v_{2}$ for different locations of the ATPD. The parameters of these distributions are taken as the average values of the corresponding parameters across the different values of $y$. Thereby, we have $\omega=2.06, \eta=1.06, \tau_{1}=$ $\tau_{2}=50 \mathrm{mph}$, and $\theta_{1}=\theta_{2}=5.86 \mathrm{mph}$.

Next, the correlations between $H, v_{1}$ and $v_{2}$ at the APTD are examined. The scatter plot in Fig. 4 based on the field data does not show evidence of dependency among $H, v_{1}$ and $v_{2}$; the points in the figure are randomly distributed in three-dimensional space with no visibly strong dependency patterns. This is reinforced by the Spearman's rank correlation coefficients in Table 2 which indicate the absence of correlations in most cases. Hence, the field data is consistent with the presumption of independence between $H, v_{1}$ and $v_{2}$ made in Section 2.4. Accordingly, the joint probability density function $f\left(H, v_{1}, v_{2}\right)$ in model 
$M 2$ is approximated by the product of the probability density functions of $H, v_{1}$, and $v_{2}$, which are denoted by $g(H), \zeta\left(v_{1}\right)$, and $q\left(v_{2}\right)$, respectively, and have the heretofore calibrated parameters.

\subsubsection{Determination and calibration of closed-form expression for $\psi$}

Based on the discussion in Section 2.4, to determine the closed-form expression for $\psi$ and calibrate it, first $\sigma^{2}$, the variance of $\varepsilon_{2}^{\text {in }}$ (or $\varepsilon_{1}^{\text {out }}$ ), is calibrated. The field data collected by an APTD located at different sites in the range $460 \mathrm{ft}$ - $1110 \mathrm{ft}$ away from the stop bar is used to generate a linear regression model $\sigma^{2}(y)=-0.10389+0.000863 * y$ which is used to estimate $\sigma^{2}$ for different values of $y$. Other regression techniques can also be used to fit the relationship between $\sigma^{2}$ and $y$. In Section 3.3.3, we additionally analyze the effect of a quadratic regression model for $\sigma^{2}(y)$.

As discussed in Section 2.4, the approximate formulation (maximum error equal to 0.01) developed by Lin (1989) is used to represent the complementary probability of $\psi$, as illustrated in Equation (20):

$$
\bar{\psi}=P(\varepsilon \leq-\Gamma)=P\left(\frac{\varepsilon}{\sqrt{2} \sigma} \leq \frac{-\Gamma}{\sqrt{2} \sigma}\right)=\frac{1}{2} \exp \left(-0.717 \frac{-\Gamma}{\sqrt{2} \sigma}-0.416\left(\frac{-\Gamma}{\sqrt{2} \sigma}\right)^{2}\right)
$$

where $\Gamma=H-2.5+\frac{y}{v_{2}}-\frac{y}{v_{1}}$. Note that $\sigma$ is calculated in Equation (20) based on the value of $y$ using the calibrated regression model.

\subsubsection{Implementation of solution search process}

Substituting the expressions for $f\left(H, v_{1}, v_{2}\right)$ and $\psi$ into $M 2$, we obtain model $M 3$, which is used to implement the solution search process.

$$
\begin{array}{cc}
M 3 & \frac{y-5.5 v_{85}}{\mathrm{v}_{85} \bar{H}} \iiint_{H-2.5+\frac{y}{v_{2}}-\frac{y}{v_{1}} \geq 0} g(H) \zeta\left(v_{1}\right) q\left(v_{2}\right) d v_{1} d v_{2} d H \\
\text { Max } & \frac{\iiint_{H-2.5+\frac{y}{v_{2}}-\frac{y}{v_{1}} \geq 0}\left(1-\psi\left(H, v_{1}, v_{2}\right)\right) g(H) \zeta\left(v_{1}\right) q\left(v_{2}\right) d v_{1} d v_{2} d H}{\iiint_{S\left(v_{1}, v_{2}, H\right)}\left(1-\psi\left(H, v_{1}, v_{2}\right)\right) g(H) \zeta\left(v_{1}\right) q\left(v_{2}\right) d v_{1} d v_{2} d H} \geq(1-\beta) \\
\text { s.t. } \quad \iiint_{H-2.5+\frac{y}{v_{2}}-\frac{y}{v_{1}} \geq 0} \psi\left(H, v_{i}, v_{j}\right) g(H) \zeta\left(v_{1}\right) q\left(v_{2}\right) d v_{1} d v_{2} d H & \frac{1}{1-\iiint_{S\left(v_{1}, v_{2}, H\right)}\left(1-\psi\left(H, v_{1}, v_{2}\right)\right) g(H) \zeta\left(v_{1}\right) q\left(v_{2}\right) d v_{1} d v_{2} d H} \leq \alpha \\
& 1100 \geq y \geq 460 \\
& v_{1}, v_{2}, H \text { are random variables. } s\left(v_{1}, v_{2}, H\right) \text { represents the feasible range of } v_{1}, v_{2}, H
\end{array}
$$

The key steps of the search process are as follows. Instead of using zero and positive infinity as the lower and upper bounds of $y$, respectively, in model $M 3$, this study first reduces the search range to 460ft $1100 \mathrm{ft}$ based on the collected data, which is adequate for the numerical experiments. Next, the feasible region for $M 3$ is determined using the prediction efficiency and safety constraints in Equations (22) and 
(23), respectively, based on a three-step procedure: (i) Calculate the left hand sides of Equations (22) and (23) as $y$ is varied from $460 \mathrm{ft}$ to $1100 \mathrm{ft}$ in increments of $10 \mathrm{ft}$, using a numerical integral technique to determine the triple integral; we use the software Matlab to do so; (ii) Determine if a location $y$ is feasible for the prediction efficiency-related threshold $\beta$ varying from 0.1 to 0.4 with increments of 0.05 , and the prediction safety-related threshold $\alpha$ varying from 0.01 to 0.05 in increments of 0.002 ; (iii) Find the intersections of the feasible regions of the prediction efficiency and safety constraints to identify the feasible region of $M 3$ under different prediction efficiency and safety requirements. Note that substantially smaller threshold values are assigned to the prediction safety constraint than to the prediction efficiency constraint in the experiments as traffic safety usually has significantly higher priority in dilemma zone protection systems. However, as stated earlier, $\beta$ and $\alpha$ can be user-defined, and the traffic engineer can choose to reduce $\beta$ and increase $\alpha$, if desired, in a field application. Finally, the optimal locations to deploy the APTD under different prediction efficiency and safety requirements are determined using the Golden-Section algorithm based on the identified feasible regions. The results and insights from the experiments are discussed next.

\subsection{Results and insights}

\subsubsection{Optimal solutions}

Table 3 illustrates the feasible ranges to deploy the advance detector and the local optimal solutions for $M 3$ for different values of the thresholds $\beta$ and $\alpha$, under the linear regression model for $\sigma^{2}(y)$ discussed in Section 3.2.2. These feasible ranges are determined through the trade-offs between the prediction efficiency and safety constraints; hence, there are combinations of $\beta$ and $\alpha$ for which no feasible solutions exist as shown in the table. The table indicates that the feasible ranges increase as the prediction efficiency and safety requirements are relaxed (that is, as the values of thresholds $\beta$ and $\alpha$ increase). Further, in the study experiments, the optimal location to deploy the APTD is at the upper bound of the computed feasible range in each case, as shown in Table 3. In summary, the mathematical formulation and solution method proposed in Section 2 enable the quantitative evaluation of the prediction efficiency and prediction safety, thereby providing the ability to identify the feasible ranges for $y$ and its optimal locations, under different thresholds.

Next, we analyze the observation that the optimal solutions are located at the upper bounds of the computed feasible ranges in the study experiments. For the collected field data, the calibrated nonlinear objective function (21) turns out to be a monotonically increasing function of $y$ over the feasible range, leading to the optimal solution occurring at the upper bound of the corresponding feasible range. However, due to the variability in traffic conditions across different intersections, this monotonicity does not necessarily hold in general. Hence, this observation cannot be generalized to other road intersections. 
Table 3 also provides several important insights for practice. Even with the relaxed constraints on efficiency (90\%; corresponding to $\beta=0.1$ ), no feasible region exists when we aim to protect $95 \%$ percent of the time (that is, $\alpha=0.05$ ). The first feasible region starts at about the $70 \%$ efficiency level. Even at the $70 \%$ efficiency and $95 \%$ safety levels, we can locate the detector at a maximum of 520ft from the stop bar. This implies that if the detector at this intersection approach is located $520 \mathrm{ft}$ away, it is expected to not terminate the green phase, when it can be safely terminated, close to $30 \%$ of the time (that is, after factoring that the green phase is terminated unsafely about $5 \%$ of the time). In typical field implementations of the green termination system, the detector is placed around $750 \mathrm{ft}-1000 \mathrm{ft}$ from the stop bar. From Table 3, it can be seen that if the detector is located at $770 \mathrm{ft}$, we are operating at only $60 \%$ efficiency and $95 \%$ safety, implying that green phase will not be terminated nearly $40 \%$ of the time when opportunities exist to do so safely.

\subsubsection{Formulation of $P(E)$}

As discussed in Section 2.2 after the formulation of $P(E)$ through Equation (11), the likelihood that a dilemma zone is empty is determined by the unfolding traffic conditions rather than the location of the APTD. As stated there, the collected field data is used here to calculate $P(E)$ using Equation (11) for different values of $y$ to validate the independence of $P(E)$ from $y$. Fig. 5 plots the relationship between $P(E)$ and $y$; the mean and variance of the calculated $P(E)$ with respect to $y$, under the linear regression model for $\sigma^{2}(y)$, are 0.25 and $4.38 \mathrm{E}-05$, respectively. The small variance indicates that the $P(E)$ estimated by Equation (11) is not perceptibly impacted by the location of the APTD. This is consistent with reality, and validates that Equation (11) is a reasonable formulation to determine the probability of an empty dilemma zone. It also validates the applicability of our assumptions related to the prediction error $\varepsilon$ whose distribution is used to determine $P(E)$ as illustrated by Equations (8)-(11).

\subsubsection{Variance of the prediction error, $\sigma^{2}(y)$}

Next, following the discussion in Sections 2.4 and 3.2.2, the effect of the prediction error $\varepsilon$ on the optimal location of the APTD is analyzed by performing sensitivity analyses from two perspectives: (i) increasing the variance of $\varepsilon$, and (ii) applying different regression models to estimate the variance of $\varepsilon$.

Using the linear regression model discussed in Section 3.2.2, we first explore how the optimal location of the APTD changes when the variance of $\varepsilon$ is artificially increased. This is done by increasing the coefficient of $y$ in the model $\left(\sigma^{2}(y)=-0.10389+w * 0.000863 y\right)$ by multiplying to it a weight $w$ varying from 1.1 to 2.9 in increments of 0.2 or 0.4 . Then, the corresponding change in the optimal location of the APTD is determined. Fig. 6 illustrates the associated results by plotting the relationship between the optimal location of $y$ and $\alpha$. The prediction safety-related threshold $\alpha$ is limited to the range $0.064-0.09$ as feasible regions do not exist for lower $\alpha$ values in this experiment, and correspondingly, the prediction 
efficiency-related threshold $\beta$ is in the range 0.3-0.4. In Fig. 6, for each value of $\alpha$, the optimal location is closer to the intersection (that is, the optimal $y$ decreases) as the prediction error increases (that is, as $w$ increases). This is consistent with the notion that reducing the optimal $y$ can reduce the opportunities for increased prediction error, thereby enhancing the robustness of the green termination algorithms. Further, we note that as $w$ increases beyond some value, the largest possible optimal $y$ also decreases as indicated by the two sets of graphs in Fig. 6, with the second graph being to the lower right side of the other graph, for $\mathrm{w}=2.3$ and beyond. By contrast, for each given $w$ (that is, when the relationship between $y$ and the prediction error is fixed), the optimal location moves further away from the intersection (optimal $y$ increases) as the prediction safety requirement is relaxed (that is, as $\alpha$ increases). In summary, the optimal location is influenced by the dependency of the prediction error on the location of the advance detector.

Next, using the collected field data set used to generate the linear regression model (used in the study experiments heretofore), a quadratic regression model, $\sigma^{2}(y)=0.633733-0.00113 y+$ $1.27 \mathrm{E}-06 y^{2}$, is generated to analyze whether different models for $\sigma^{2}(y)$ to estimate the variance of $\varepsilon$ affect the optimal location of $y$. The corresponding feasible ranges and optimal solutions under different prediction efficiency and safety requirements are shown in Table 4.

The results from Tables 3 and 4 corresponding to the linear and quadratic models, respectively, are compared. Both indicate that as the prediction efficiency and safety constraints are relaxed, the feasible region to locate the APTD increases and the corresponding optimal solutions are located further away from the intersection at the upper bounds of their feasible ranges. However, the linear and quadratic models result in different optimal solutions. For example, for $\alpha$ up to 0.044 , no feasible solutions exist when the quadratic regression model is used to estimate the variance of the prediction error at different locations, but some feasible solutions exist under the linear model. Also, for $\alpha$ of 0.046 or larger, the optimal location of the APTD under the quadratic regression model is further away from the intersection compared to that under the linear model. These observations can be explained using Figs. 7 and 8 which illustrate the prediction efficiency $p_{e}$ and prediction safety $p_{s}$, respectively, when $\sigma^{2}(y)$ is represented by linear (labeled $\mathrm{L}$ in the figures) and quadratic (labeled $Q$ in the figures) regression models.

Figs. 7 and 8 illustrate that both $p_{e}$ and $p_{s}$ deteriorate under the linear model as well as under almost all quadratic model cases as the APTD is located further away from the intersection. Fig. 7 further shows that $p_{e}$ is almost identical for the linear and quadratic cases over the whole range of $\mathrm{y}$. However, Fig. 8 indicates that $p_{s}$ in the linear case is larger than that under the quadratic case in the APTD location range 460ft-570ft. Correspondingly, when $p_{s}$ is greater than 0.955 (that is, $\alpha<0.046$ ), the feasible region exists for the linear case, but not the quadratic case as seen in Tables 3 and 4 . In the APTD location range $570 \mathrm{ft}-970 \mathrm{ft}, p_{s}$ is larger under the quadratic case. Hence, the feasible region is larger under the quadratic 
model, and correspondingly the optimal APTD locations are further away from the intersection. Beyond 970ft, $p_{S}$ is below the acceptable bound for the prediction safety constraint (that is, $\alpha \geq 0.05$ ), and hence that APTD range is not addressed.

In summary, the proposed methodology is sensitive to the variance of the prediction error $\varepsilon$ as well as the regression model structure which describes the relationship between the variance of the prediction error $\varepsilon$ and the location of the APTD. When the traffic flow has higher variability, the prediction error $\varepsilon$ may have a larger variance; in such scenarios, the optimal location of the APTD may shift closer to the intersection.

\section{Concluding comments}

As dilemma zones exist widely at signalized intersections in the United States, traffic controllers deploy an advance detector at an intersection which collects traffic data near the dilemma zone to provide support for dilemma zone protection methods such as green termination algorithms. However, currently, the deployment of advance detectors is determined mostly based on engineering experience rather than rigorous analytical models that provide bounds on the prediction errors to aid the traffic engineer. Thereby, the existing approach can entail inefficiencies due to the adoption of conservative dilemma zone boundaries. In this context, some prior studies have recognized that the location of the advance detector significantly impacts the efficiency of dilemma zone protection systems, but systematic analytical studies do not exist that can identify the optimal location of the APTD by factoring the various problem characteristics.

This study proposes a nonlinear optimization model to determine the optimal location of the APTD by factoring the need for operational efficiency and safety in the development of green termination algorithms for high-speed isolated signalized intersections. The associated objective manifests as the need to maximize the expected number of times to predict an empty dilemma zone during a look-ahead time period so as to maximize the number of opportunities that the green phase can be terminated both safely and efficiently. Due to the problem characteristics, the location of the APTD is constrained by prediction efficiency and prediction safety requirements, which serve as constraints in the proposed optimization model. The solution method combines a line search algorithm with numerical integration techniques to determine the optimal location of the APTD. The practical applicability and the implementation of the proposed approach are illustrated using field data collected at a high-speed isolated signalized intersection in Lincoln, Nebraska. Numerical experiments are also used to validate the problem methodology and provide insights on the role of user-provided inputs. Thereby, inputs by traffic engineers on efficiency- and safety-related thresholds based on the characteristics at an intersection can be seamlessly integrated into the proposed analytical methodology. 
The numerical experiments illustrate that when the efficiency- and safety-related thresholds are relaxed, the optimal location to deploy the APTD shifts further away from the intersection, and vice versa. The optimal solution occurs at the upper bound of the computed feasible region which varies with the values of the prediction efficiency and safety thresholds. Further, the optimal solution is affected by the relationship between the prediction error $\varepsilon$ and the location of the APTD. Calibrated linear and quadratic regression models for the variance of the prediction error as a function of the APTD location lead to different optimal solutions. This highlights the need to robustly calibrate the relationship between the prediction error and the location of the ATPD using field data.

From a practice perspective, the study confirms the potential concerns related to the performance efficiency of green termination systems using a point detector, as typical field implementations locate the detector $750 \mathrm{ft}-1000 \mathrm{ft}$ from the stop bar. For the study intersection, it is observed that with the detector located at $770 \mathrm{ft}$, the prediction efficiency is barely $60 \%$ under a $95 \%$ safety level, implying that the green termination system may miss nearly $40 \%$ of the potential opportunities to terminate the green due to an incorrect prediction that the dilemma zone is occupied. In this context, the proposed methodology not only identifies the optimal location, but also provides its feasible range subject to user-specified efficiency-and safety-related thresholds.

In this study, overtaking of vehicles downstream of the APTD location is not considered in the modeling. However, overtaking is possible between the time instant a vehicle passes the advance detector and the time instant it enters the dilemma zone, especially in multi-lane situations under certain traffic conditions. This represents a future research consideration in the determination of the optimal APTD location.

\section{References}

Bazaraa, M. S., Sherali, H. D., Shetty, C.M., 1993. Nonlinear programming theory and algorithms, 2rd Edition. John Wiley \& Sons, New York.

Bonneson, J.A., McCoy, P.T., Moen, B.A., 1994. Traffic detector design and evaluation guidelines. Report No. : TRP-02-31-93. Nebraska Department of Roads, Lincoln, Nebraska.

Bonneson, J.A, Middleton, D., Zimmerman, K., Charara, H., Abbas, M., 2002. Intelligent detection-control system for rural signalized intersections. Report No. FHWA/TX-03/4022-2. Texas Department of Transportation, Austin, Texas.

Chang, M.S., Messer, C.J., Santiago, A.J., 1985. Timing traffic signal change intervals based on driver behavior. Transportation Research Record 1027, 20-30. 
Gerlough, D.L., Huber, M.J., 1975. Traffic flow theory: a monograph. Special Report 165, Transportation Research Board, National Research Council, Washington D.C.

Gerstner, T., Griebel, M., 1998. Numerical integration using sparse grids. Numerical Algorithms 18, 209232.

Herman R., Olson, P.L., Rothery, R.W., 1963. Problem of the amber signal light. Traffic Engineering and Control 5, 298-304.

Kronborg, P., Davidsson, F., Edholm, J., 1997. SOS- self optimising signal control development and field trials of the SOS algorithm for self optimizing signal control at isolated intersections. TFK Report, TFK- Transport Research Institute, Stockholm, Sweden.

Kumar P., 2010. Probability distributions and estimation of Ali-Mikhail-Haq Copula, Applied Mathematical Sciences 4 (14), 657- 666.

Lin, J.T. (1989). Approximating the normal tail probability and its inverse for use on a pocket-calculator. Applied Statistics 38, 69-70.

Liu, Y., Chang, G.L., Tao, R., Hicks, T., Tabacek, E., 2007. Empirical observations of dynamic dilemma zones at signalized intersections, Transportation Research Record 2035, 122-133.

May, A.D., 1968. Clearance interval at flashing systems. Highway Research Record 221, Washington D.C., 41-71.

Newell, G.F., 1989. Theory of highway traffic signals. Report No.: UCB-ITS-RR-89-7. Institute of Transportation Studies. University of California Berkley.

Olson, P.O., Rothery, R.D., 1962. Response to amber phase of traffic signals. Bulletin 330, Highway Research Board, 40-51.

Parsonson, P.S., 1978. Signalization of high speed, isolated intersections. Transportation Research Record $681,34-42$.

Press, W.H., Farrar, G.R., 1990. Recursive stratified sampling for multidimensional monte carlo integration. Computers in Physics 4, 190-195.

Sharma, A., Bullock, D., and Peeta, S., 2006. Limitations of simultaneous gap out logic. Transportation Research Record 1978, 42-48.

Sharma, A., Bullock, D., and Peeta, S., 2007. Recasting dilemma zone design as a marginal cost and benefit problem. Transportation Research Record 2035, 88-96. 
Sharma, A., Bullock, D., and Peeta, S., 2011a. Estimating dilemma zone hazard function at high speed isolated intersection. Transportation Research Part C: Emerging Technologies 19 (3), 400-412.

Sharma, A., Bullock, D., Velipasalar, S., Casares, M., Schmitz, J., Burnett, N., 2011b. Improving safety and mobility at high speed intersections with innovations in sensor technology. TRB $90^{\text {th }}$ Annual Meeting Compendium of Papers, (Recommended for publication).

Webster, F., and Ellson, P., 1965. Traffic signals for high speed roads, Road Research Laboratory, Road Research Technical Paper No. 74, HMSO, London.

Zegeer, C., 1977. Effectiveness of green-extension systems at high-speed intersections. Research Report 472, Bureau of Highways, Kentucky Department of Transportation, Lexington, Kentucky.

Zhang, G.H., Wan, Y.H., Wei, H, Chen, Y.Y., 2007. Examining headway distribution models using urban Freeway loop event data, Transportation Research Record 1999, 141-149.

Zimmerman, K., Bonneson, J.A, Middleton D., Abbas, M.M, 2003. Improved detection and control system for isolated high-speed signalized intersections. Transportation Research Record 1856, 212-219.

Zimmerman, K., 2007. Development of a second generation detection-control system for safer operation of high-speed signalized intersections. Report No. TRB-NCHRP-115, Transportation Research Board. 


\section{Tables}

Table 1

Empty dilemma zone prediction and the operation of dilemma zone protection systems

\begin{tabular}{||l||c|c||}
\hline & Dilemma zone occupied & Dilemma zone empty \\
\hline $\begin{array}{l}\text { Dilemma zone is predicted to be } \\
\text { occupied }\end{array}$ & Correct prediction. & $\begin{array}{c}\text { Error event } e^{e} . \\
\text { Impacts efficiency. }\end{array}$ \\
\hline $\begin{array}{l}\text { Dilemma zone is predicted to be } \\
\text { empty }\end{array}$ & $\begin{array}{c}\text { Error event } e^{s} . \\
\text { Impacts safety. }\end{array}$ & Correct prediction. \\
\hline
\end{tabular}


Table 2

Results of Correlation Test for $H, v_{1}$ and $v_{2}$

\begin{tabular}{|c|c|c|c|c|c|c|c|}
\hline$y$ & $n$ & $r\left(v_{1}, v_{2}\right)$ & $t$-test & $r\left(v_{1}, H\right)$ & $t$-test & $r\left(v_{2}, H\right)$ & $t$-test \\
\hline 475 & 97 & -0.0116 & -0.1132 & 0.3402 & 3.5256 & 0.2680 & 2.7108 \\
\hline 500 & 97 & -0.0829 & -0.8111 & 0.1820 & 1.8044 & 0.1399 & 1.3771 \\
\hline 525 & 97 & -0.0702 & -0.6856 & 0.1739 & 1.7214 & 0.2175 & 2.1715 \\
\hline 550 & 96 & 0.0137 & 0.1323 & 0.1095 & 1.0676 & 0.2695 & 2.7131 \\
\hline 575 & 89 & 0.0153 & 0.1423 & 0.2292 & 2.1968 & 0.121297 & 1.1398 \\
\hline 600 & 88 & 0.0792 & 0.7366 & 0.1705 & 1.6043 & 0.162675 & 1.5290 \\
\hline 625 & 92 & 0.2271 & 2.2120 & 0.1726 & 1.6621 & 0.2822 & 2.7903 \\
\hline 650 & 96 & 0.2258 & 2.2474 & 0.1508 & 1.4791 & 0.1875 & 1.8511 \\
\hline 675 & 96 & 0.1847 & 1.8218 & 0.3500 & 3.6228 & 0.0399 & 0.3868 \\
\hline 700 & 95 & 0.1490 & 1.4532 & -0.1357 & -1.3208 & 0.1944 & 1.9112 \\
\hline 725 & 95 & 0.1207 & 1.1729 & 0.1578 & 1.5412 & -0.0495 & -0.4776 \\
\hline 750 & 94 & 0.2177 & 2.1394 & 0.1337 & 1.2941 & -0.1328 & -1.2851 \\
\hline 775 & 93 & 0.0997 & 0.9560 & 0.1353 & 1.3027 & 0.0136 & 0.1294 \\
\hline 800 & 90 & -0.1168 & -1.1035 & 0.1785 & 1.7016 & -0.0184 & -0.1726 \\
\hline 825 & 86 & 0.0020 & 0.0182 & 0.2430 & 2.2964 & 0.0160 & 0.1462 \\
\hline 850 & 84 & 0.1384 & 1.2655 & 0.2352 & 2.1914 & 0.0895 & 0.8139 \\
\hline 875 & 79 & 0.1447 & 1.2834 & 0.0133 & 0.1166 & 0.0248 & 0.2175 \\
\hline 900 & 76 & 0.0640 & 0.5517 & 0.3931 & 3.6778 & 0.0763 & 0.6586 \\
\hline 925 & 74 & -0.2709 & -2.3875 & 0.1740 & 1.4993 & 0.1003 & 0.8552 \\
\hline 950 & 68 & -0.0843 & -0.6875 & 0.0285 & 0.2314 & -0.0648 & -0.5274 \\
\hline 975 & 64 & -0.1676 & -1.3389 & -0.2142 & -1.7266 & 0.2124 & 1.7116 \\
\hline 1000 & 49 & -0.1267 & -0.8759 & 0.0615 & 0.4226 & 0.0392 & 0.2688 \\
\hline 1025 & 43 & 0.0529 & 0.3389 & -0.0636 & -0.4079 & 0.2241 & 1.4724 \\
\hline 1050 & 40 & -0.1592 & -0.9946 & 0.0407 & 0.2512 & 0.0792 & 0.4896 \\
\hline 1075 & 34 & 0.1117 & 0.6358 & 0.2376 & 1.3836 & -0.2382 & -1.3874 \\
\hline 1100 & 32 & -0.0235 & -0.1285 & 0.0704 & 0.3865 & 0.0304 & 0.1667 \\
\hline \multirow{2}{*}{\multicolumn{5}{|c|}{$\begin{array}{l}t \text {-test: significance level: } 0.05 ; 1.98<t(.975, \mathrm{n})<2 \\
\mathrm{H}_{0}: \text { there is no association between the two variables } \\
\mathrm{H}_{\mathrm{a}} \text { : there is an association between the two variables }\end{array}$}} & & \multicolumn{2}{|l|}{ Confirm $\mathrm{H}_{\mathrm{a}}$} \\
\hline & & & & & & \multicolumn{2}{|c|}{ Confirm $\mathrm{H}_{0}$} \\
\hline
\end{tabular}

$r(*, *)$ : Spearman's rank correlation coefficient; $n$ : the number of observations at a $y$. 
Table 3

Feasible range and optimal solution (using linear regression model for $\sigma^{2}$ )

\begin{tabular}{||l||l|l|l|l|l|l||}
\hline \hline$(\alpha, \beta)$ & $0.01-0.04$ & 0.042 & 0.044 & 0.046 & 0.048 & 0.05 \\
\hline \hline $0.1-0.25$ & $\mathrm{~N}$ & $\mathrm{~N}$ & $\mathrm{~N}$ & $\mathrm{~N}$ & $\mathrm{~N}$ & $\mathrm{~N}$ \\
\hline 0.3 & $\mathrm{~N}$ & {$[460,490 *]$} & {$[460,520 *]$} & {$[460,520 *]$} & {$[460,520 *]$} & {$[460,520 *]$} \\
\hline 0.35 & $\mathrm{~N}$ & {$[460,490 *]$} & {$[460,540 *]$} & {$[460,600 *]$} & {$[460,670 *]$} & {$[460,730 *]$} \\
\hline 0.4 & $\mathrm{~N}$ & {$[460,490 *]$} & {$[460,540 *]$} & {$[460,600 *]$} & {$[460,670 *]$} & {$[460,770 *]$} \\
\hline \multicolumn{7}{|c|}{$\sigma^{2}(y)=-0.10389+0.000863 * y$} \\
\hline
\end{tabular}

$\left[a, b^{*}\right]$ : feasible range from $a$ to $b^{*} ; b^{*}$ is the optimal solution; $\mathrm{N}$ : no feasible solution. 
Table 4

Feasible range and optimal solution (using quadratic regression model for $\sigma^{2}$ )

\begin{tabular}{||r||l|l|l|l|l|l||}
\hline$(\alpha, \beta)$ & $0.01-0.04$ & \multicolumn{1}{|c|}{0.042} & 0.044 & 0.046 & 0.048 & \multicolumn{1}{|c|}{0.05} \\
\hline \hline $0.1 \sim 0.25$ & $\mathrm{~N}$ & $\mathrm{~N}$ & $\mathrm{~N}$ & $\mathrm{~N}$ & $\mathrm{~N}$ & $\mathrm{~N}$ \\
\hline 0.3 & $\mathrm{~N}$ & $\mathrm{~N}$ & $\mathrm{~N}$ & {$[490,530 *]$} & {$[460,530 *]$} & {$[460,530 *]$} \\
\hline 0.35 & $\mathrm{~N}$ & $\mathrm{~N}$ & $\mathrm{~N}$ & {$[490,620 *]$} & {$[460,730 *]$} & {$[460,730 *]$} \\
\hline 0.4 & $\mathrm{~N}$ & $\mathrm{~N}$ & $\mathrm{~N}$ & {$[490,620 *]$} & {$[460,770 *]$} & {$[460,860 *]$} \\
\hline \multicolumn{7}{|r|}{$\sigma^{2}(y)=0.633733-0.00113 * y+1.27 \mathrm{E}-06 * y^{2}$} \\
\hline
\end{tabular}

$\left[a, b^{*}\right]$ : feasible range from $a$ to $b^{*} ; b^{*}$ is the optimal solution; $\mathrm{N}$ : no feasible solution. 


\section{Figures}

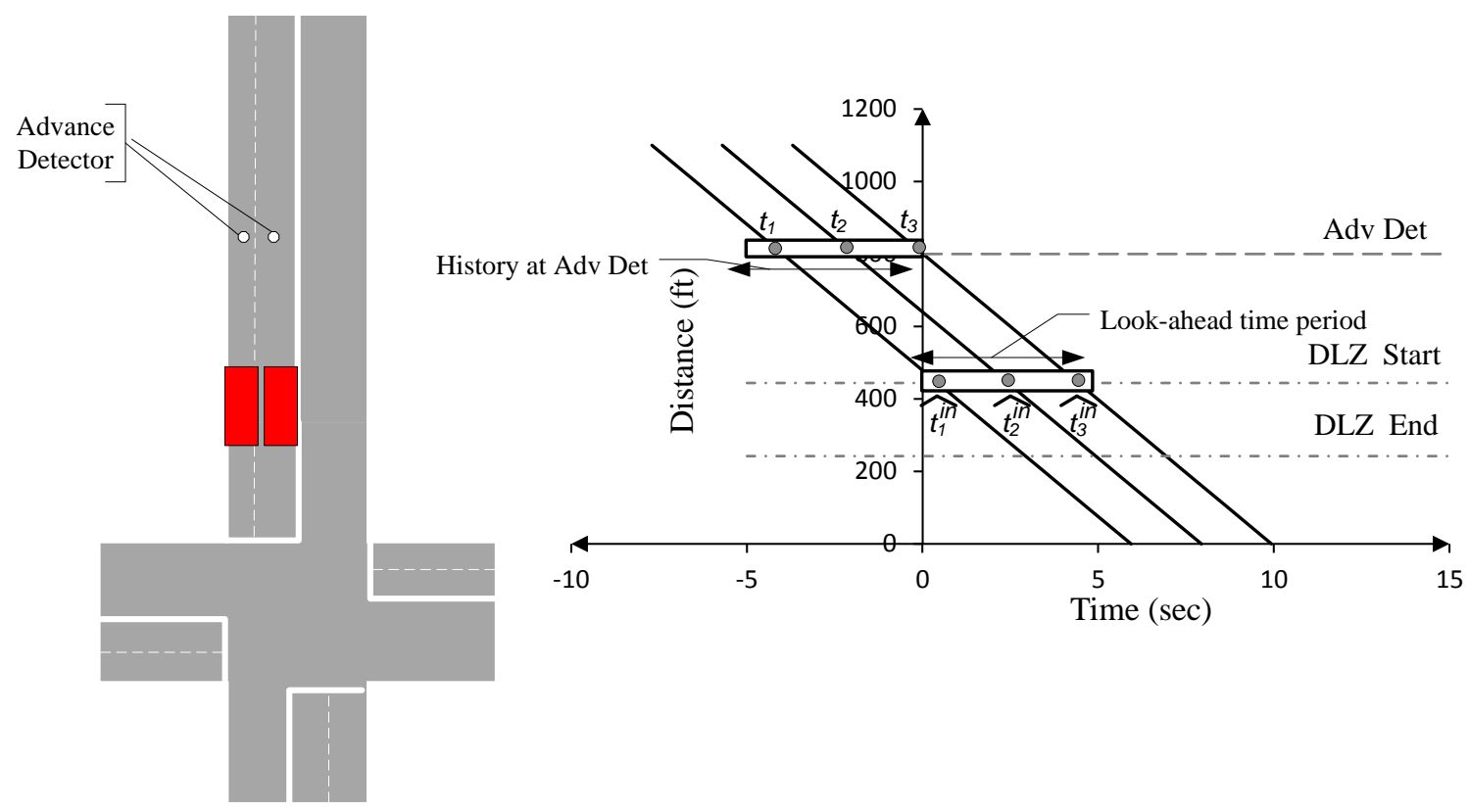

Fig. 1 Illustration of green termination logic 


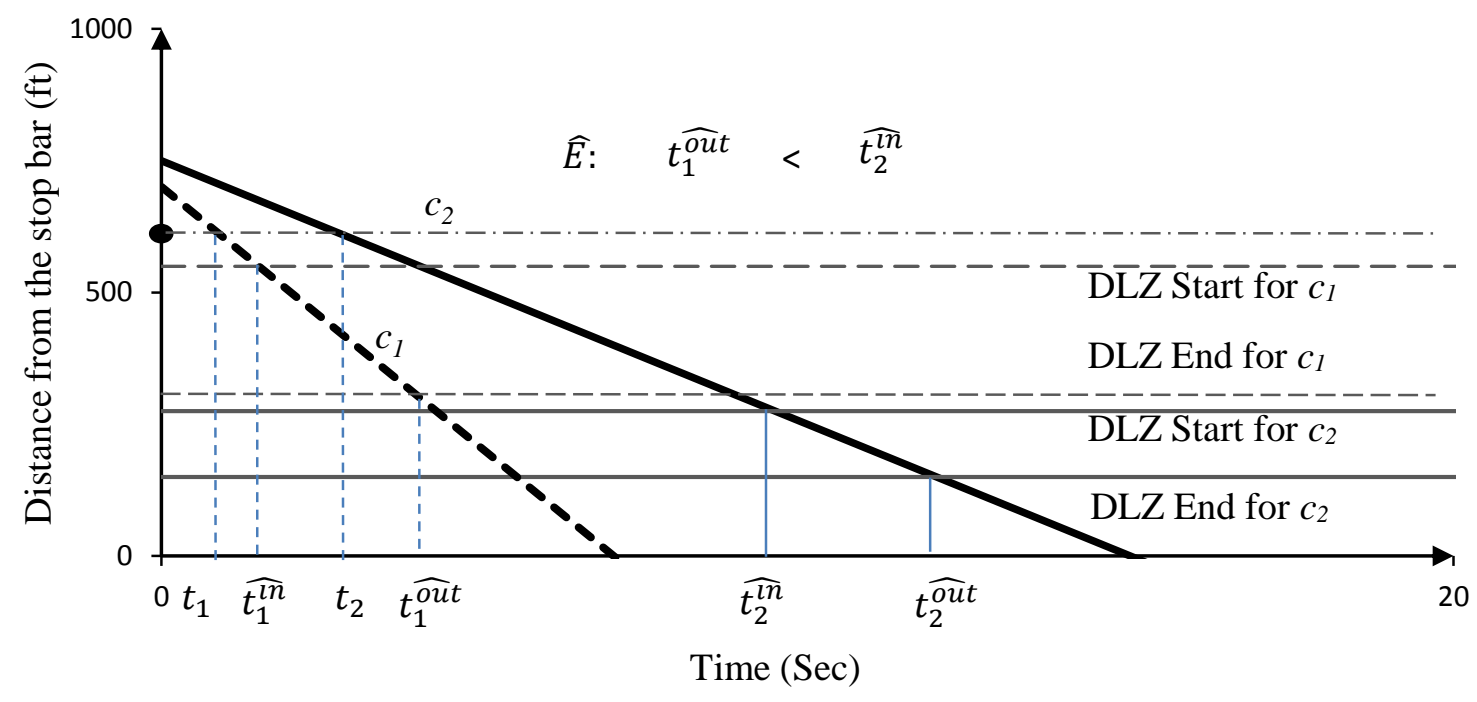

Fig. 2. Illustration of empty dilemma zone prediction 


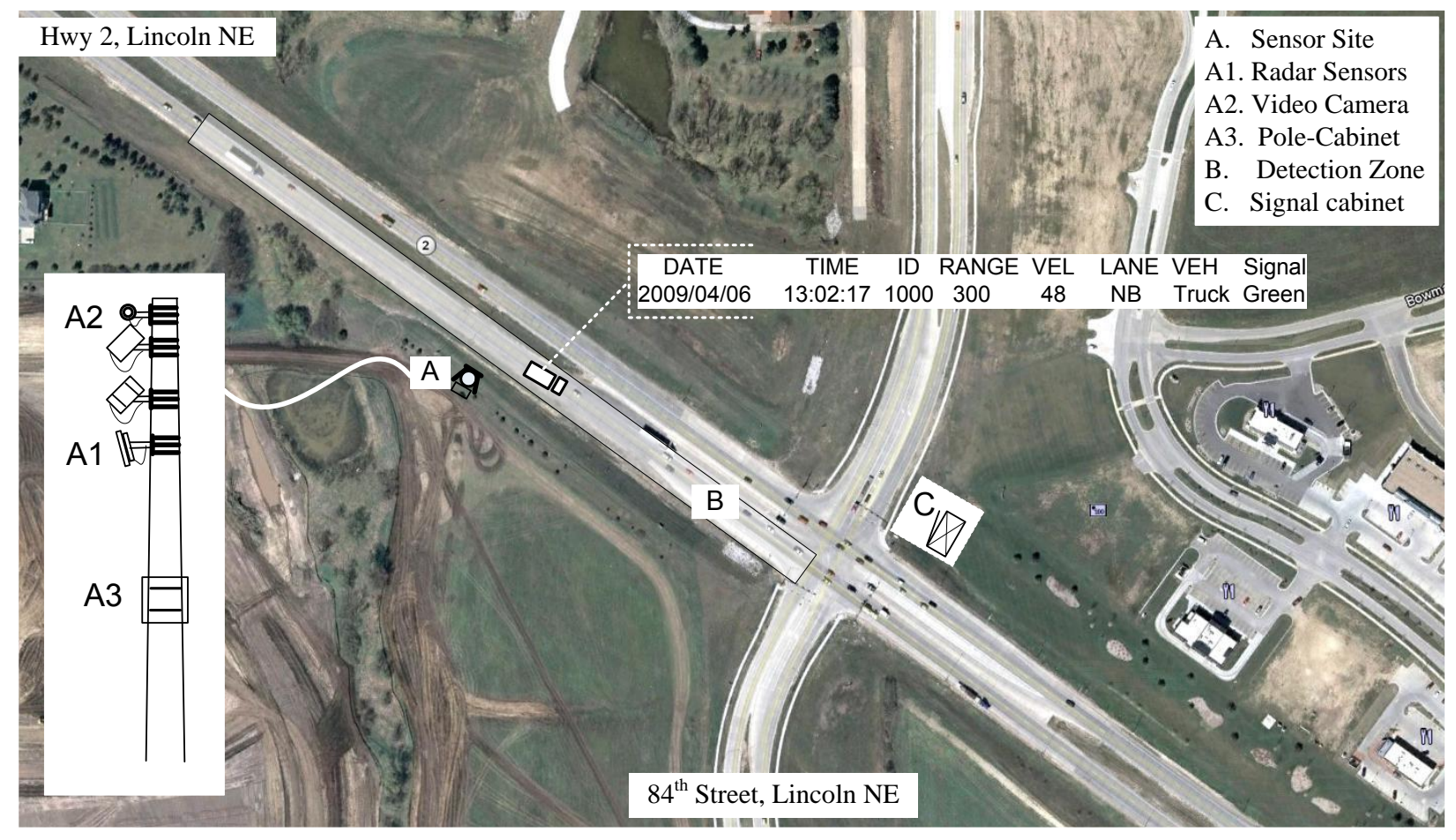

Fig. 3. The Highway 2 and $84^{\text {th }}$ Street intersection testbed, Lincoln, NE 

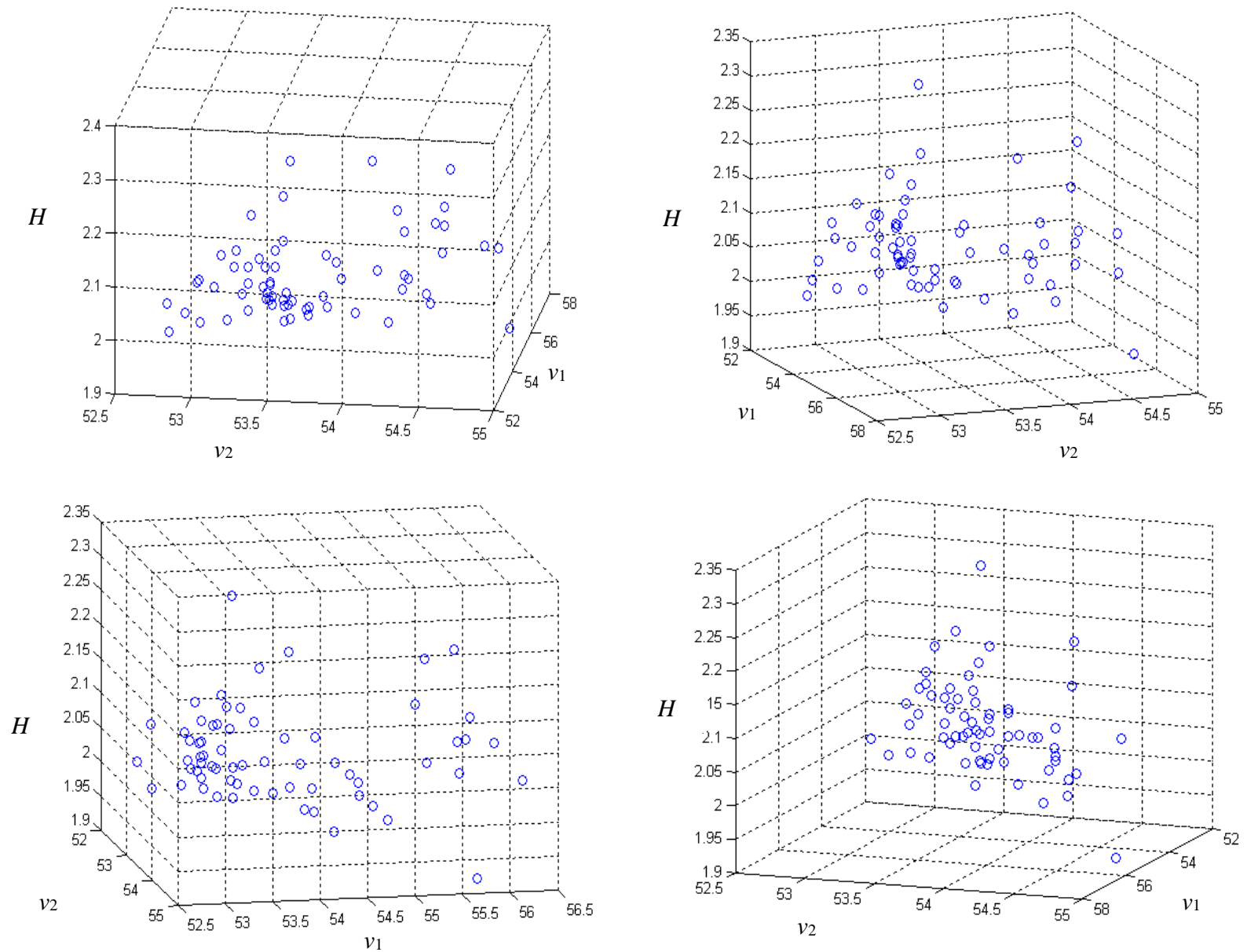

Fig. 4. Scatter plots of $v_{1}, v_{2}$ and $H$ 


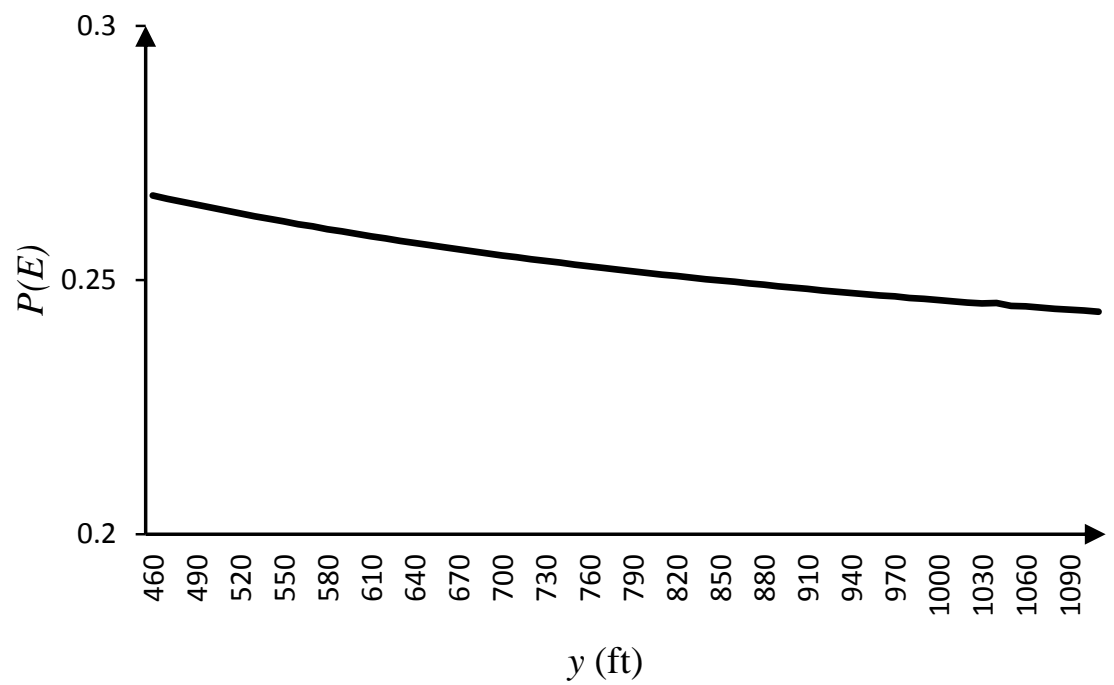

Fig. 5. $P(E)$ for different locations of APTD 


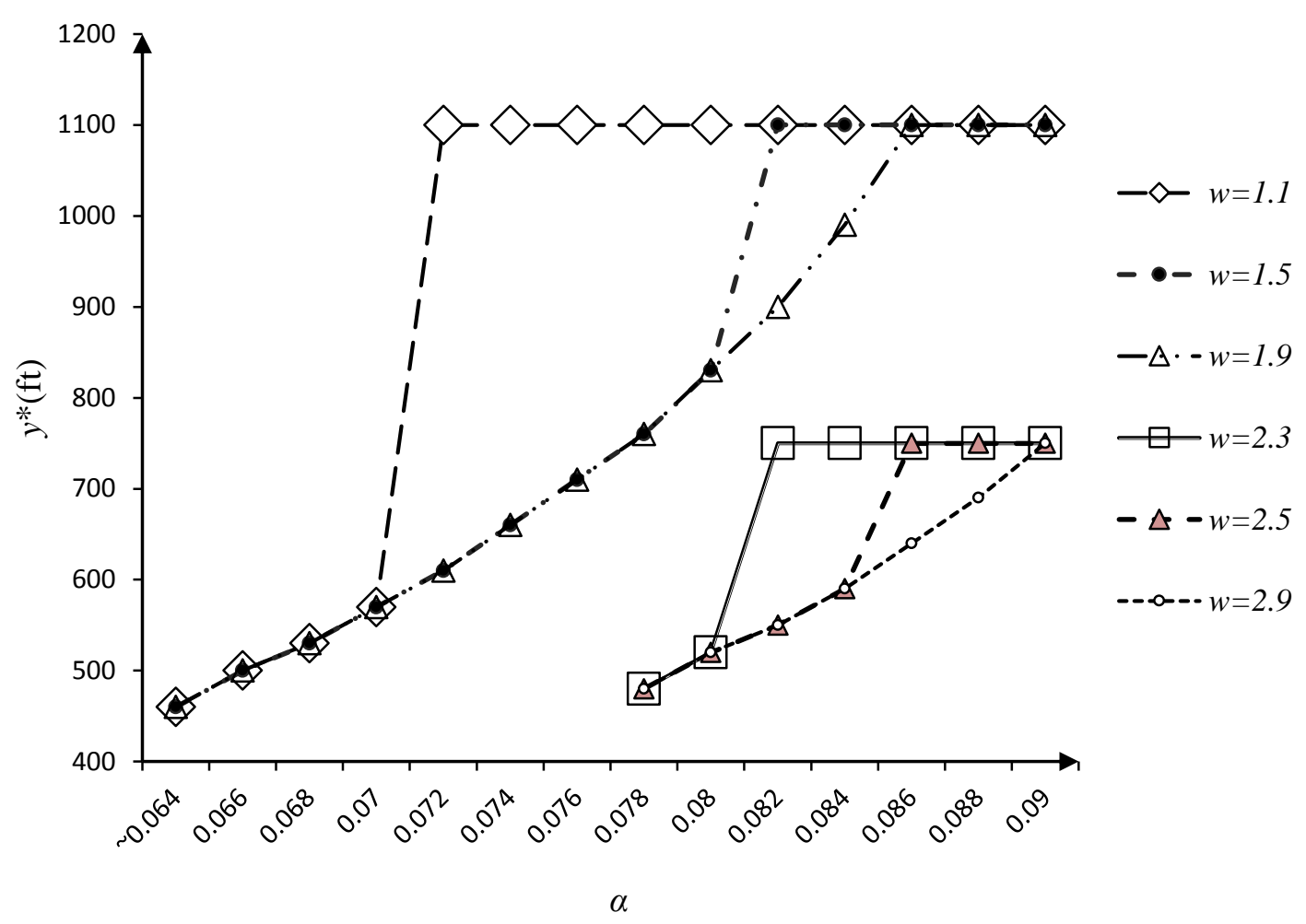

Fig. 6. The effect of the value of $\sigma^{2}(y)$ at study site on the optimal location of the APTD 


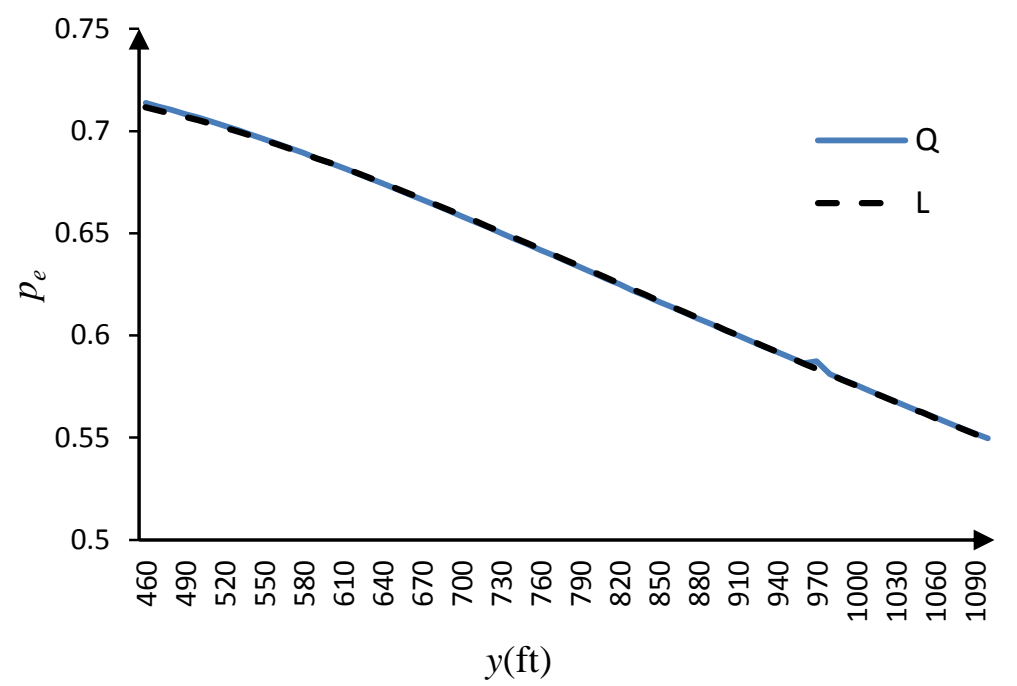

Fig. 7. Prediction efficiency $p_{e}=P(\hat{E} \mid E)$ 


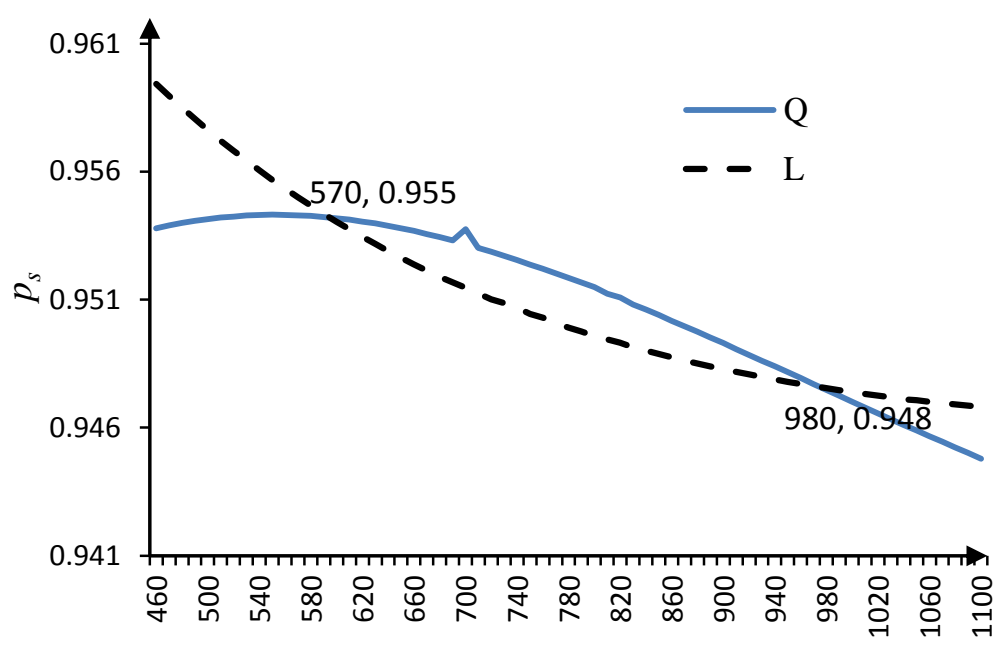

$y(\mathrm{ft})$

Fig. 8. Prediction safety $p_{s}=P(\overline{\hat{E}} \mid \bar{E})$ 